\title{
Meconio: vehiculo de biomarcadores de exposición a sustancias psicoactivas en gestantes
}

\author{
Sandra Manrique López¹, Sonia Puentes García1, \\ Milena Orjuela Velasco ${ }^{1}$ \\ 1. Estudiantes del Programa de Bacteriología y Laboratorio Clínico, Facultad de Ciencias de la Salud, \\ Universidad Colegio Mayor de Cundinamarca, Bogotá, Colombia. \\ Correspondencia: sanmmalo@yahoo.com
}

Recibido: 12-06-2007 / Aceptado: 28-06-2007

\begin{abstract}
Resumen
La población infantil expuesta prenatalmente a drogas de abuso constituye un problema médico-social. Una forma útil de abordar este problema consiste en analizar matrices biológicas, como el meconio, que acumula sustancias psicoactivas o sus metabolitos. Su determinación de la exposición fetal se ha centrado en el análisis de sangre de cordón u orina materna o neonatal. El meconio es inerte y de fácil obtención, acumula sustancias psicoactivas o sus metabolitos desde la semana 12 de gestación donde quedan fosilizados hasta el nacimiento. Puede constituir un instrumento muy importante para investigar la exposición fetal a distintas drogas de abuso. Es necesario conocer el grado de exposición fetal para contribuir al diagnóstico y al tratamiento oportuno de patologías que por falta de manifestaciones clínicas relevantes, pasan inadvertidas y requieren la implementación de pruebas que utilicen matrices biológicas confiables.
\end{abstract}

Palabras Clave: cocaína, etanol, marihuana, meconio, retardo del crecimiento fetal, síndrome alcohólico fetal, síndrome de abstinencia neonatal.

\begin{abstract}
Children exposed to drugs in a prenatal way are a medical and social problem. A useful way to study this problem is by analyzing biological matrices, such as the meconium, which stores psychoactive substances or their metabolites. To determine its fetal exposure the most common system is the analysis of blood from the umbilical cord, urine from the mother, or urine from the new born. Meconium is inert and it is easily obtainable. It stores psychoactive substances or its metabolites from week 12 of gestation and they are fossilized in it until birth. It can be a very important instrument to investigate fetal exposure to several drugs of abuse. It is necessary to know the degree of fetal exposure to contribute to the prognosis and timely pathology treatment which, due to the lack of clinical relevant manifestations, can be overlooked and require the implementation of tests that use reliable biological matrices.
\end{abstract}

Key words: cocaine, etanol, fetal growth retardation, fetal alcohol syndrome, marijuana, meconium, neonatal abstinence syndrome. 


\section{Introducción}

El consumo de sustancias psicoactivas (SPA) por la gestante constituye uno de los principales problemas de salud publica, produce alteraciones en el feto y recién nacido (RN) que van desde Retardo del Crecimiento Fetal (RCIU) hasta Sufrimiento Fetal Agudo (SFA), Síndrome de Abstinencia Neonatal (SAN), Síndrome Alcohólico Fetal (SAF) o la muerte. Estas complicaciones hacen indispensable una valoración eficaz y la implementación de pruebas que utilicen matrices biológicas confiables para determinar el consumo de SPA en las gestantes y de esta manera contribuir en el diagnóstico y tratamiento oportuno de la madre y el neonato. El análisis de meconio permite conocer el grado de exposición fetal a estas sustancias, ya que por su capacidad de fosilización, carácter acumulativo e iniciar su formación finalizando el primer trimestre de gestación actúa como vehículo de biomarcadores de exposición.

\section{Desarrollo embrionario}

En el proceso de desarrollo embrionario el cigoto experimenta una serie de divisiones mitóticas, posteriormente se implanta en el endometrio uterino y se inicia la formación de las capas extraembrionarias: saco amniótico, saco vitelino, alantoides y corion leve y frondoso (1,2,3). A partir de la $5^{\text {a }}$ semana de gestación el amnios, alantoides y saco vitelino forman el cordón umbilical que constituye la unión entre el embrión y la placenta $(2,3)$.

La placenta continúa su formación hasta las semanas $18^{\mathrm{a}}$ y $20^{\mathrm{a}}$, pero crece durante todo el embarazo. Sus principales funciones son: protectora (inmunológica y contra traumatismos), metabólica (intercambio de gases, nutrientes y electrólitos) y endocrina (síntesis de hormonas como progesterona, estradiol y HCG) $(3,4)$. Cabe resaltar que a partir del $4^{\circ}$ mes de desarrollo, el grosor de esta membrana se reduce debido a que el tejido conectivo disminuye, el citotrofoblasto desaparece y el sincitiotrofoblasto se adelgaza; esta reducción facilita el paso de SPA ocasionando efectos letales al feto $(4,5)$.
Los primeros 3 meses de gestación son claves en el adecuado desarrollo del nuevo ser, ya que durante este período se forman su cerebro, órganos internos y extremidades. Por esta razón una gestante no debe consumir ningún tipo de sustancias que alteren el normal desarro1lo fetal. En el $2^{\circ}$ trimestre hay crecimiento corporal y maduración de las estructuras desarrolladas a lo largo del 1 er trimestre, y en el $3^{\text {er }}$ trimestre hay desarrollo completo de órganos y sistemas $(2,3)$.

El sistema digestivo (eje para la formación del meconio) se forma a partir de un intestino primitivo dividido en tres partes: anterior, medio y posterior. El duodeno hace parte del intestino medio y al cabo de la $5^{\mathrm{a}}$ y $6^{\mathrm{a}}$ semana de gestación, su luz se torna más pequeña debido a la proliferación de células epiteliales, una parte de ellas se descaman y constituyen uno de los componentes del meconio (2).

Por otra parte, el hígado, la vesícula biliar y los conductos biliares surgen como una evaginación ventral de la porción caudal del intestino anterior. A partir de la $6^{\mathrm{a}}$ semana de gestación se inicia en el hígado la hematopoyesis y posteriormente el catabolismo de la hemoglobina, alrededor de la $12^{\mathrm{a}}$ semana comienza la formación de bilis por los hepatocitos y penetra en el duodeno a través del colédoco confiriéndole al meconio color verde oscuro (2).

Durante el embarazo, el feto deglute líquido amniótico (LA) que contiene proteínas y otras sustancias, las cuales se filtran y permanecen en la parte posterior del intestino, mientras que el líquido amniótico (LA) es liberado nuevamente al espacio uterino cuando el feto orina (2). Este ciclo es un proceso de reciclaje que ocurre aproximadamente cada 3 horas y mantiene el LA en condiciones adecuadas durante los nueve meses de gestación $(2,3)$.

\section{Meconio}

El meconio es la primera deposición del RN, es expulsado entre 12 y 48 horas después del nacimiento; su textura es viscosa, pegajosa y de color verde negruzco. 
Está formado por agua, lípidos, proteínas, esteroles, precursores del colesterol, ácidos grasos libres, productos de la deglución del LA, células epiteliales, bilis y secreciones intestinales (6). Las ventajas que ofrece el uso del meconio están dadas por su fácil obtención (proceso no invasivo), se acumula desde el segundo trimestre del embarazo y es metabólicamente inerte, por lo que una vez los tóxicos o sus metabolitos llegan, permanecen fosilizados en altas concentraciones, permitiendo su estudio posterior $(6,7)$.

Los SPA y sus metabolitos alcanzan el meconio por varias vías: difusión por transporte sanguíneo, deglución del LA y excreción en el tracto intestinal a través de la bilis después de su metabolismo hepático (6). Estudios que emplearon esta muestra para detectar cocaína en RN indican que tiene una sensibilidad del $87 \%$, una especificidad del $100 \%$, un $32 \%$ de falsos negativos y $0 \%$ de falsos positivos, cifras superiores a las obtenidas en orina materna $(6,7)$.

Tradicionalmente los esfuerzos para determinar la exposición fetal se han centrado en el análisis de cordón $\mathrm{u}$ orina de la madre o el neonato (8). Sin embargo, estas muestras reflejan consumo de unos días previos al parto y en el caso de la orina materna presenta sensibilidad del $37 \%$ y de un $30-60 \%$ de falsos negativos $(7,9)$; además se utiliza principalmente para determinar benzoilecgonina -principal metabolito urinario de cocaínasi está presente en una concentración superior a $150 \mathrm{ng} /$ $\mathrm{mL}(10)$.

\section{Sustancias psicoactivas y efectos de exposición prenatal}

Las SPA pueden ser consideradas biomarcadores ya que son compuestos químicos que se encuentran en el organismo como ellos mismos o como metabolitos u otras formas conjugadas de los mismos (11). Los biomarcadores son conocidos también como determinantes o indicadores biológicos de exposición y se pueden medir en el organismo empleando técnicas tanto invasivas como no invasivas.
Los efectos del consumo de SPA durante la gestación marcan un aumento notable en las tasas de morbilidad materno-fetal, dependen del tipo de droga, dosis, politoxicomanía, tiempo de exposición fetal, período gestacional y su capacidad para atravesar la barrera placentaria (12-15). Se ha comprobado que la mayor parte de drogas de abuso atraviesan la placenta, causando sobre-estimulación de los sistemas de neurotransmisión del feto, lo cual puede producir anomalías en los patrones de proliferación, diferenciación y migración neuronal, que son controlados parcialmente por estos sistemas de neuro-transmisión, así como alteraciones secundarias en las vías compensatorias que modulan el estado de bienestar y contribuyen a déficits conductuales posteriores (16-18).

Según estadísticas de la Secretaria Distrital de Salud (SDS) el consumo de SPA aumentó un 36\% en el 2006 respecto al año anterior, las mujeres en edad fértil (1640 años) consumen en orden de importancia alcohol, marihuana y cocaína $(19,20)$. A continuación se mencionan aspectos generales de las SPA de mayor consumo, como su composición química, farmacocinética y los posibles efectos en el desarrollo prenatal y postnatal.

El Etanol $\left(\mathrm{CH}_{3} \mathrm{CH}_{2} \mathrm{OH}\right)$ tiene un efecto estimulante y depresor sobre el sistema nerviosos central (SNC) (21), es catalogado como una de las drogas con alto poder teratogénico $(22,23)$ debido a su bajo peso molecular, baja polaridad y afinidad lipofílica (24), propiedades que le permiten atravesar la barrera placentaria exponiendo al feto a concentraciones iguales, o incluso superiores, a las que la madre tiene en sangre (25).

Farmacocinética: El etanol se absorbe por vía oral en el tubo digestivo, alcanza su concentración máxima a los 30-60 minutos en la corteza cerebral y una absorción total en seis horas. Luego, pasa a la sangre y se distribuye por todos los tejidos (15). La eliminación comienza inmediatamente después de su administración y sólo pequeñas cantidades se desechan sin modificar por el riñón, sistema respiratorio y glándulas sudoríparas (26). 
El metabolismo se realiza en el hígado por la vía enzima alcohol deshidrogenasa (ALDH) que da lugar a la producción de acetaldehído, producto tóxico que rápidamente es transformado, por oxidación, en acetato, el cual incrementa la producción de lactato y ácidos grasos, que interfirieren en las funciones hepáticas al disminuir el ácido cítrico y la oxidación de ácidos grasos $(21,24)$.

El etanol se adhiere a los ácidos grasos del organismo y crea esteres etil de ácidos grasos (EEAGs), que son metabolitos no oxidativos (27), se acumulan en meconio y permanecen fosilizados hasta el nacimiento, confiriéndoles capacidad como biomarcadores de exposición. Además, el hallazgo de más de $520 \mathrm{ng} / \mathrm{g}$ en meconio indica consumo materno (28-30).

La descomposición de los metabolitos del alcohol es lenta en el cuerpo inmaduro del feto y esto hace que los niveles permanezcan elevados por más tiempo (25), considerándose como un compuesto citotóxico que genera daño neuronal producido por cambios irreversibles en la fluidez de las membranas sinápticas, desacoplamiento de la fosforilación oxidativa en la mitocondria, excitotoxicidad inducida por hiperactivación del receptor de neuraminidasa (NMDA), entrada masiva de calcio intracelular en situaciones de abstinencia $y$ aumento de la fragilidad lisosomal en las células pancreáticas $(31,32)$.

Los EEAG que se encuentran en mayor proporción son: Ácidos ésteres etil oleico, palmitoleico, palmítico, esteárico, linoleico, heptadecanoico y araquidónico. En algunos estudios, se ha detectado en el meconio y sangre de cordón umbilical etil linoleico y palmítico, lo cual permite un avance significativo en el diagnóstico y tratamiento del RN con exposición prenatal (29).

Efectos en el desarrollo prenatal y postnatal: durante la organogénesis el etanol, por su acción teratogénica, destruye las células de la línea media anterior del disco embrionario y produce deficiencia de estructuras craneofaciales. En el periodo fetal afecta órganos en desarrollo como los ojos, el sistema respiratorio y SNC. Además, se generan trastornos postnatales de conducta, dificultades en el aprendizaje y bajo coeficiente intelectual (25).

El RCIU, retraso mental y malformaciones craneofaciales (microcefalia, labios delgados con hundimiento del labio superior, nariz chata y en "trompeta" con baja inserción del puente nasal) componen el Síndrome Alcohólico Fetal (SAF), característico en hijos de madres con antecedentes de alcoholismo durante el embarazo. Además, el alcohol eleva la frecuencia cardiaca y la presión arterial, perjudicando la salud de la madre y el feto, adicionalmente hay más probabilidades de un parto prematuro o desprendimiento de la placenta. Es de resaltar que todo tipo de consumo representa un riesgo ya que no existe un nivel seguro de ingesta que evite el desarrollo del síndrome $(21,33)$.

La cocaína (Benzoilmetilecgonina, eritroxilina o metilester de benzoilecgonina) es un alcaloide estimulante del SNC (34).

Farmacocinética: la forma más común es el clorhidrato de cocaína, su consumo se realiza por vía inhalatoria e intravenosa que permite un rápido transporte al cerebro $(34,35)$. La inhalación de cocaína produce efectos a los 20-30 minutos, su distribución (1.5-2 L/Kg) es amplia y llega a todo el organismo (35). Se metaboliza rápidamente por hidrólisis enzimática y produce metabolitos lipofílicos, de liberación lenta $(36,37)$, que pueden ser inactivos (benzoilecgonina y ecgonina) (37) o activos (norcocaína) (35). La eliminación es rápida, se excreta de $1-5 \%$ por la orina, sin cambios (34). La benzoilecgonina es el metabolito que se detecta en orina de 3-4 días después del último consumo (36).

En presencia de etanol la cocaína es transesterificada por esterasas hepáticas a etilcocaína o cocaetileno y se incrementa la N-demetilación a Norcocaína (metabolito activo). El etanol inhibe la actividad de la metilesterasa, disminuyendo la hidrólisis a benzoilecgonina (metabolito inactivo); el cocaetileno posee actividad farmacológica y tóxica, fundamentalmente a nivel cardiaco y hepático, y posee una vida media 2 ó 3 veces más larga que la cocaína (38). 
Efectos en el desarrollo prenatal y postnatal: el principal mecanismo que utiliza la cocaína es la vasoconstricción y taquicardia materna-fetal (aumento de contractilidad uterina y disminución de flujo úteroplacentario) (39), la disminución del flujo ocasiona reducción en el transporte de oxígeno y de nutrientes, causando RCIU (40-42).

Se destacan principalmente las malformaciones (15) genitourinarias, esqueléticas respiratorias y alteraciones neurológicas, estas últimas ocasionadas por traspaso de la barrera hematoencefálica, ingresa al SNC, bloquea la captación de catecolaminas $(42,43)$, generando irritabilidad y anomalías conductuales $(41,42,44)$. Aumenta la morbilidad y mortalidad infantil, especialmente perinatal y complicaciones obstétricas (desprendimiento prematuro de placenta, parto pretérmino y placenta previa) $(15,45)$.

Por otro lado, la asociación con el alcohol forma un nuevo compuesto a nivel hepático (cocaetileno) que atraviesa la placenta (46), es biológicamente activo y ha sido detectado en LA y meconio, el cual aumenta el desarrollo del SAN y del SAF, que potencian los rasgos clínicos de ambos síndromes, especialmente los craneofaciales y algunas malformaciones mayores: cardiacas, renales y cerebrales entre otras (47).

La marihuana (cannabis) actúa como estimulante sobre el SNC. Químicamente contiene más de 400 sustancias, de las cuales la más conocida es el 9-deltatetrahidrocannabinol o 9-D-THC, causante de los principales efectos de la droga $(26,48,49)$.

Farmacocinética: cuando la marihuana es fumada, un 50\% del 9-D-THC pasa a la sangre y se une fuertemente a proteínas transportadoras (21). El 9-D-THC se volatiliza rápidamente y es absorbido por el pulmón. Se metaboliza en el hígado, en menor medida en el SNC y en la mucosa del intestino delgado y se elimina en dos terceras partes por vía enterohepática y el restante por riñón, donde puede ser detectada hasta por espacio de 3 días (uso casual) a 4 semanas (uso diario fuerte) $(48,49)$.
Las membranas de las células nerviosas en el cerebro, órgano blanco más importante de la acción perinatal, contienen receptores que captan el 9-D-THC; una vez estos se unen, ocurren reacciones celulares que causan daños irreparables como pérdida parcial de la memoria y problemas de aprendizaje $(50,51)$.

Efectos en el desarrollo prenatal y postnatal: los cannabinoides causan modificación en todas las fases de la reproducción y el desarrollo. Las acciones producidas por estas sustancias ocurren en tres etapas:

1. Exposición preconcepcional: altera gametogénesis e inhibe la fertilización por modificación en el eje hipotálamo-adenohipófisis-gónada y en presencia de altas concentraciones de 9-D-THC no progresa la división celular del cigoto. 2. Exposición prenatal: altera la organogénesis, el desarrollo de la cavidad bucal y causa déficit de maduración del sistema visual. 3 . Exposición Postnatal: durante la lactancia causa retraso en el desarrollo, bajo peso corporal, alteración en el eje adeno-hipófisis-gónada, déficit en el aprendizaje y retraso de reflejos visuales $(15,50,52)$.

Las situaciones en que se debe sospechar de abuso de drogas son: madre con historia de alto riesgo (déficit en control prenatal, embarazo en adolescencia, desnutrición, problemas psicológicos, abruptio placentae, parto prematuro) y $\mathrm{RN}$ con RCIU, microcefalia, malformaciones craneofaciales o SAN (19). La técnica más sencilla para determinar si un RN proviene de una madre consumidora es evaluar anamnesis y encuesta dirigida, sin embargo, estos datos son poco fiables para establecer una impresión diagnostica $(12,19)$ que contribuya al tratamiento oportuno ante manifestaciones de SAN. En la Tabla 1 se muestran algunas manifestaciones $(15,25)$.

En ausencia de síntomas que acompañan estos síndromes y una historia de consumo materno positivo, es necesario investigar y evidenciar el uso de SPA por medio de técnicas sensibles y especificas que permitan identificar la sustancia o sus metabolitos (19). 
Tabla 1. Valoración SAN.

\begin{tabular}{ll}
\hline \multicolumn{1}{c}{ Sistema Afectado } & \multicolumn{1}{c}{ Signos y síntomas } \\
\hline SNC & $\begin{array}{l}\text { Irritabilidad, temblores, hiperactividad, hipertonía, llanto agudo, } \\
\text { mioclonias y convulsiones. }\end{array}$ \\
\hline Sistema Digestivo & Regurgitaciones, vómito, diarrea y ansiedad. \\
\hline Alteraciones vegetativas & $\begin{array}{l}\text { Sudoración, hipersecresión de mucosas, fiebre, erupciones } \\
\text { cutáneas. }\end{array}$ \\
\hline $\begin{array}{l}\text { Alteraciones } \\
\text { cardiorespiratorias }\end{array}$ & Taquipnea, congestión nasal y taquicardia. \\
\hline
\end{tabular}

Tabla 2. Propiedades químicas y su relación con el paso por la barrera placentaria

\begin{tabular}{|c|c|c|c|c|}
\hline \multirow[t]{2}{*}{ SUSTANCIA } & \multirow{2}{*}{$\begin{array}{c}\text { PESO } \\
\text { MOLECULAR }\end{array}$} & \multirow{2}{*}{$\begin{array}{c}\text { LIPO } 0 \\
\text { HIDROFILIDAD }\end{array}$} & \multicolumn{2}{|c|}{$\begin{array}{l}\text { TRANSFERENCIA } \\
\text { MATERNO-FETAL }\end{array}$} \\
\hline & & & Potencial & $\begin{array}{c}\text { Tasa } \\
\text { (in vitro) }\end{array}$ \\
\hline ETANOL & $46-07$ & Lipofílica & Alto & $\begin{array}{c}35-95 \\
\text { umol/mL/min }\end{array}$ \\
\hline COCAÍNA & 303.45 & Hidrofílica leve & Moderado & $\begin{array}{c}0.16 \mathrm{ug} / \mathrm{mL} / \mathrm{min} \\
\text { Cocaetileno } \\
0.009 \mathrm{ug} / \mathrm{mL} / \mathrm{min}\end{array}$ \\
\hline MARIHUANA & 314.45 & Muy lippofílica & Muy alto & $26.96 \mathrm{umol} / \mathrm{L}$ \\
\hline
\end{tabular}

\section{Transferencia placentaria}

El transporte placentario de sustancias se establece alrededor de la $5^{\text {a }}$ semana de vida embrionaria, pero antes de formarse la placenta cualquier sustancia puede actuar directamente sobre las células embrionarias o sobre órganos maternos, alterando de forma indirecta el desarrollo fetal $(12,19)$. Sin embargo, la gestante tiene la capacidad de desintoxicar su cuerpo al descomponer sustancias extrañas, a diferencia del feto, quien posee una mucosa gastrointestinal altamente permeable y un sistema enzimático inmaduro que evita el metabolismo normal (12).

El metabolismo de SPA se lleva a cabo en la vellosidades coriónicas donde origina metabolitos tóxicos, que por sus propiedades químicas como bajo peso molecular $(<990 \mathrm{Da})$, baja polaridad y afinidad lipofílica
(Tabla 2) atraviesan la barrera placentaria con mayor facilidad por medio de mecanismos como difusión facilitada $(12,45,53)$.

A medida que la placenta madura, el espesor del epitelio trofoblástico disminuye y aumenta la superficie de contacto, lo cual conlleva a que se eleve la velocidad de difusión de las sustancias cuando la placenta tiene mayor edad (53). Por otra parte la eliminación de SPA en el compartimiento fetal se realiza por difusión hacia el compartimiento materno, pero muchos metabolitos quedan en la porción fetal hasta que el riñón adquiere capacidad de eliminar drogas circulantes al LA (12,53). Esta capacidad de eliminación reducida y la circulación fetal a través de la vena umbilical, que alcanza directamente su corazón y cerebro, sin paso previo por el hígado, implica una disminución en el 
metabolismo de las drogas, haciendo que el efecto de SPA en el feto sea mayor (53).

A manera de síntesis, y teniendo como base las fuentes de consulta, se puede anotar que la exposición prenatal a SPA genera mayor efecto durante el $1^{\mathrm{er}}$ trimestre de gestación, las características físico-químicas de cada sustancia les confieren capacidad para atravesar las vellosidades coriónicas de la placenta y ocasionar desarrollo anormal. La mayoría de las anomalías se originan durante la organogénesis pero antes o después de esta etapa pueden producirse defectos, de manera que ningún período de desarrollo es completamente seguro, ya que cada sistema orgánico puede tener una etapa o más de susceptibilidad.

La valoración del $\mathrm{RN}$ ofrece información sobre las condiciones de vida intrauterina y evalúa la capacidad de adaptación al ambiente externo. La ausencia de alteraciones evidentes junto con una historia clínica dudosa no siempre es un indicador del estado clínico del $\mathrm{RN}$, por lo tanto se debe contar con métodos de detección más fiables para confirmar la exposición a SPA.

El meconio ofrece mayor sensibilidad y especificidad en la detección de EEAG, Benzoilecgonina, Ecgonina, Norcocaína, Cocaetileno y 9-D-THC (54-57) con respecto a otras matrices biológicas como orina, sangre materna y de cordón o pelo (58). Para que el meconio pueda ser considerado como un biomarcador de exposición fetal a SPA se necesita desarrollar y validar la metodología necesaria para medir cuantitativamente estas sustancias.

De otro lado, la población infantil expuesta prenatalmente a drogas de abuso constituye un problema médico-social que bajo una intervención temprana proporcionaría el desarrollo de medidas preventivas. Consideramos que el estudio de SPA en meconio es un objetivo de investigación que permitirá ampliar la aplicación clínica actual que por el momento está limitada a otras sustancias. NOV

\section{Referencias}

1. Kaloðlu C, Gürsoy E, Onarlioðlu B. Early maternal changes contributing to the formation of the chorioallantoic and yolk sac placentas in rat: a morphological study. Anat Histol Embryol. 2003;32:200-206.

2. A. C. Enders A, Carter A. What Can Comparative Studies of Placental Structure Tell Us?-A Review Placenta 2004; 25:S3-S9.

3. Burton G, Jauniaux E, Charnock-Jones D. Human Early Placental Development: Potential Rolesof the Endometrial Glands. Placenta. 2007;21:S64-S69.

4. Weissgerber T, Wolfe L. Physiological adaptation in early human pregnancy: adaptation to balance maternal-fetal demands. Appl Physiol Nutr Metab. 2006;31:1-11

5. Myren M, Mose T, Mathiesen L, Knudsen L. The human placenta-An alternative for studying foetal exposure. Toxicol In Vitro. 2007; 7: [Epub ahead of print].

6. Ortega J., Carrizo D, Ferris J, Peinador M, Grimalt J. Meconium and neurotoxicans. Revista Española De Pediatría. 2004,60:291-296.

7. Mur A, García O, López N. Toxicidad de la cocaína en el recién nacido. Detección y prevalencia. An Esp Pediatr 2002;56:241-246

8. Ostrea E, Knapp D, Tannenbaum L, Ostrea A, Romero A, Salari V, Ager J. Estimates of illicit drug use during pregnancy by maternal interview, hair analysis, and meconium analysis. J Pediatr 2001;138:344-348.

9. Doris J, Meguid V, Thomas M, Blatt S, Eckenrode J. Prenatal cocaine exposure and child welfare outcomes. Child Maltreat. 2006;11:326-337.

10. Lewis D, Moore C, Leikin J, Koller A. Meconium analysis for cocaine: a validation study and comparison with paired urine analysis. J Anal Toxicology. 1995;19:148-150.

11. Kapusta N, Plener P, Schmid R, Thau K, Walter H, Lesch O. Multiple substance use among young males. Pharmacol Biochem Behav. 2007;86:306-311.

12. Schuetze P, Eiden R, Coles C. Prenatal cocaine and other substance exposure: effects on infant autonomic regulation at 7 months of age. Dev Psychobiol. 2007;49:276-89

13. Shankaran S, Lester B, Das A, Bauer C, Bada H, Lagasse L, Higgins R. Impact of maternal substance use during pregnancy on childhood outcome. Semin Fetal Neonatal Med. 2007; 12:143-50.

14. Periódico El Tiempo. El Consumo De Alcohol, Marihuana, Cocaína Y Basuco Está 'Disparado’ En Bogotá. Julio 31 De 2006.

15. Gauthier T, Manar M, Brown L. Is maternal alcohol use a risk factor for early-onset sepsis in premature newborns? Alcohol. 2004;33:139-145.

16. Nelson S, Lerner E, Needlman R, Salvator A, Singer L. Cocaine, anemia, and neurodevelopmental outcomes in children: a longitudinal study. J Dev Behav Pediatr. 2004;25:1-9.

17. Herlenius E, Lagercrantz H. Neurotransmitters and neuromodulators during early development. Early Hum Dev $2001 ; 65: 21-37$.

18. Stanwood G, Levitt P. Prenatal exposure to cocaine produces unique developmental and long-term adaptive changes in dopamine D1 receptor activity and subcellular distribution. J Neurosci. 2007;27:152-157.

19. Tapia J, Ventura P. Manual de neonatología. Edt. Mediterráneo. Santiago de Chile. 2000.

20. Vigilancia Epidemiológica Del Consumo De SPA En Bogota, Estadísticas, 2001, 2004, 2005, 2006. Secretaria Distrital De Salud.

21. Velasquez. J. Farmacología y Toxicología Clínica. Ed. Panamericana 2005, 384-392. 
